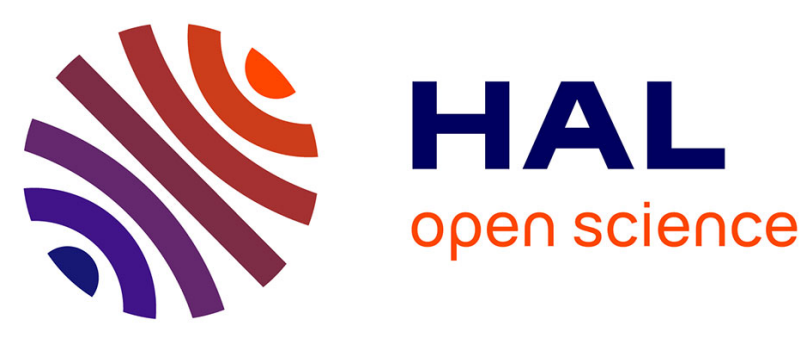

\title{
On-chip Earth spin detection
}

Thibaut Sylvestre

\section{To cite this version:}

Thibaut Sylvestre. On-chip Earth spin detection. Nature Photonics, 2020, 14, pp.341-343. hal02993795

\section{HAL Id: hal-02993795 \\ https://hal.science/hal-02993795}

Submitted on 25 Nov 2020

HAL is a multi-disciplinary open access archive for the deposit and dissemination of scientific research documents, whether they are published or not. The documents may come from teaching and research institutions in France or abroad, or from public or private research centers.
L'archive ouverte pluridisciplinaire HAL, est destinée au dépôt et à la diffusion de documents scientifiques de niveau recherche, publiés ou non, émanant des établissements d'enseignement et de recherche français ou étrangers, des laboratoires publics ou privés. 
News and Views on « Earth Rotation Measured by a Chip-Scale Ring Laser Gyroscope », by Yu-Hung Lai et al., Nature Photonics, to appear in 2020.

Thibaut Sylvestre, Research Director at CNRS, FEMTO-ST institute, University Bourgogne Franche-Comté, Besançon, France

\section{Title : On-Chip Earth Spin Detection}

\section{A monolithic chip-scale silica-on-silicon ring laser gyroscope based both on Brillouin and Sagnac effects provides sensitivity sufficient to measure sinusoidal rotations with amplitude as small as $5^{\circ} /$ hours, thus enabling the first on-chip earth rotation measurement.}

As scientists who plie our trade to photonics research and innovation, we never cease to be inspired by the exceptional creativity of truly great physicists of the past centuries whose works and ideas were or are unremittingly brilliant and revolutionary in the whole history of human thought. The pioneering works in the $19^{\text {th }}$ and $20^{\text {th }}$ centuries by French physicists, Foucault, Sagnac and Brillouin, are excellent cases in point. In 1851, by interpreting the motion of a heavy iron ball of $28 \mathrm{~kg}$ swinging from a wire of $67 \mathrm{~m}$ ( 220 feet) long at the Pantheon in Paris, Foucault proved that Earth rotates about its own axis with a $15^{\circ} /$ hours rate [1]. Such a "Foucault pendulum" always swings in the same vertical plane, but on a rotating Earth, this vertical plane slowly changes, at a rate and direction dependent on the geographic latitude of the pendulum. However, the demonstration was not sufficiently convincing for many contemporaries, which prompted Foucault to invent the spinning wheel mechanical gyroscope the following year [1] (See Figure 1a). Foucault thus presented in 1852 a device capable of maintaining an amazing rotation speed of up to 200 rotations/second, allowing the rotation to persist for 10 minutes so that observable measurements can be carried out.

In 1913, Sagnac demonstrated experimentally that it is also possible to detect rotation with an optical system that has no moving part [2]. He used a ring interferometer and showed that rotation induces a phase shift between the two counter-rotating light waves. The Sagnac effect, proportional to the speed of rotation, is related to the fact that the time taken by the two beams to travel a complete lap is different. It can be understood as resulting from the addition of velocities in special relativity. Surprisingly, what is true for these photons is also true for any mass objects regardless of their rotation speed. Consider for example two snails on a turnstile, each moving at the same constant speed but in opposite ways. The composition law for velocities in special relativity entail, in the Galilean laboratory reference frame, different speeds for the two snails, due to the different walking path from two clocks placed respectively on the back of each snail, an effect analog to the global positioning satellite system (GPS). However, the Sagnac effect turns out to be dramatically small and the original setup was far from a practical rotation rate sensor. In 1925, Michelson and Gale [3] were able to measure Earth rotation with a gigantic ring interferometer of almost $2 \mathrm{~km}$, but the Sagnac effect has remained a rarely observed physical curiosity for several decades, because it was not possible to get usable performance from a reasonably compact device. Not until the early 1960s did the first ring laser gyroscope invented [4].

Compact optical gyroscopes have then been a pursuit for more than 50 years in the search for the ultimate ultra-precise rotation measurements and inertial navigation. They are increasingly finding wide applications, particularly in industry as they are a key technology in aeronautics, naval, terrestrial, and space systems for civil and military applications. In the search for the ultimate ultra-precise rotation measurements and inertial navigation robust in any situation, both the ring laser (RLG) and the fiber optic gyroscopes (FOG) are particularly attractive for their broad performances, such as high sensitivity and stability over time, beyond of elegant operating principles [4,5]. Together with micro-electro-mechanical system (MEMS) and hemispherical quartz resonator gyroscopes (HRG), both based on the Coriolis force, they constitute four main types of mature rotation sensors used outside the laboratory [6]. 
The development of a reliable and low-cost miniaturized optical gyroscope, having a minimum detectable angular velocity of the order of $10^{\circ} / \mathrm{h}$, is a key technological challenge, which is motivating an increasing research effort [6]. Until quite recently, resonant micro-optic gyroscopes (RMOG) have been limited in performance by the high losses and large-scale optical resonators. The recent generation of monolithic ultra-low-loss waveguide and centimeter-size chip-based high-Q nonlinear resonators has started to change this situation $[7,8,9]$.

Now writing in Nature Photonics, Vahala and coworkers report an on-chip Brillouin laser gyroscope which provides combined levels of sensitivity and stability that enable measurement of the Earth's rotation rate for the first time in a photonic integrated circuit [10] (See Figure 2a for a conceptual illustration). A clever offset-counter pumping technique and drift compensation method was implemented, enabling better sensitivity to rotation than the last chip [8]. Summing up, the chip gyro uses a silica wedge resonator on a silicon chip with a $Q$ factor above 100 million and a precise diameter of $36 \mathrm{~mm}$ that perfectly matches the Brillouin frequency (6 FSRs). The micro-disk resonator is bidirectionally pumped with an amplified diode laser at $1.55 \mu \mathrm{m}$ using a tapered fiber. One pump is modulated using an acousto-optic modulator (AOM) and locked to the cavity resonance using the Pound-Drever-Hall technique, while the other pump is freely tuned using another AOM to create a pump frequency detuning. The pumps generate two counterpropagating and frequency-detuned Brillouin Stokes waves through stimulated Brillouin backscattering (SBS). Besides providing optical gain, SBS is fundamentally a low-noise coherent process that enhances the gyro sensitivity [11]. The pumps and Brillouin lasers are combined and photo-detected to measure the dual-Brillouin and pump-Brillouin beating signals. The signals are analyzed using an electrical spectrum analyzer and two frequency counters. The resonator is packaged in a small brass box with fiber connectors (See Figure 1d).

The system was installed on an automated air-bearing rotation stage for the Earth rotation measurement. When the gyroscope's axis is oriented towards North and South, the round-trip travel time of two counterrotating Brillouin laser modes is different due to the Earth rotation (See Figure $2 b$ ). This difference changes the frequency splitting of the Brillouin cavity modes allowing conversion of rotation into a frequency beat measurement of a few $\mathrm{Hz}$ only (See Figure 3 of Ref. [10]). In contrast, orienting the gyroscope axis along the East and West directions induces no rotation along the gyroscope axis. The monolithic Brillouin gyroscope has an angle random walk as low as $0.0068 / \sqrt{h}$ and bias stability $<3.6^{\circ} / \mathrm{h}$. This is particularly impressive given the fact that the Sagnac shift turns out to be extremely small as it scales down with the micro-resonator effective area. However, performances are still very far from those of state-of-art RLGs and FOGs that can now readily achieve a strategic-grade sensitivity as high as $10^{-3} \% / \mathrm{h}$ and a bias stability better than $10^{-4} \% \mathrm{~h}[5]$. Nonetheless, there is great interest in the chip-based photonic devices as these can potentially provide scalability for manufacturing and also resistance to shock and vibration in harsh environments.

With any novel work, continued progress is required. In the present work, resolution and drift compensation could be further improved as could the fundamental limitations from shot noise, Kerr nonlinearity, and Rayleigh scattering [12]. That said, a flag in the ground has been staked and the possibilities for chip-scale photonic gyroscopes are promising. A long road still lies ahead before the detection of the precession and nutation of the earth's axis or to measure exactly how much the rotation is slowing down by the moon and the sun (about 1 second every 50,000 years), as precise atomic clocks do. 
References:

1. L. Foucault, "Démonstration physique du mouvement de rotation de la terre au moyen du pendule," C. r. heb d. séances Acad. sci. Paris 32, 135-138 (1851).

2. G. Sagnac, "L'éther lumineux démontré par l'effet du vent relatif d'éther dans un interféromètre en rotation uniforme," C. r. heb d. séances Acad. sci. Paris 95, 708-710 (1913).

3. A. A. Michelson and H. G. Gale, "The effect of the Earth's rotation on the velocity of light," J. of Astrophysics 61, 401 (1925).

4. W. M. Macek and D. T. M. Davis, Jr. "Rotation rate sensing with traveling-wave ring lasers," Appl. Phys. Lett. 2, 67-68 (1963).

5. H. C. Lefevre, The Fiber-Optic Gyroscope (Artech House, 2014), Second edition.

6. C. Ciminelli, F. Dell'Olio, C. E. Campanella, and M. N. Armenise, "Photonic technologies for angular velocity sensing," Adv. Opt. Photon. 2, 370-404 (2010).

7. K.Y. Yang, D.Y. Oh, S.H Lee, Q.-F. Yang, X. Yi, B. Shen, H. Wang, and K. Vahala, "Bridging ultrahigh$Q$ devices and photonic circuits," Nature Photon 12, 297-302 (2018).

8. J. Li, M. -G. Suh, and K. Vahala, "Microresonator Brillouin gyroscope," Optica 4, 346-348 (2017).

9. W. Liang, V. S. Ilchenko, A. A. Savchenkov, E. Dale, D. Eliyahu, A. B. Matsko, and L. Maleki, "Resonant microphotonic gyroscope," Optica 4, 114-117 (2017).

10. Y.-H. Lai, M.-G. Suh, Y.-K. Lu, B. Shen, Q.-F. Yang-, H. Wang, J. Li, S. H. Lee, K. Y. Yang, K. Vahala, "Earth Rotation Measured by a Chip-Scale Ring Laser Gyroscope, Nature Phot., to appear in 2020.

11. F. Zarinetchi, S. P. Smith, and S. Ezekiel, "Stimulated Brillouin fiber-optic laser gyroscope," Opt. Lett. 16, 229-231 (1991).

12. A. B. Matsko, W. Liang, A. A. Savchenkov, , V. S. Ilchenko, and L. Maleki, "Fundamental limitations of sensitivity of whispering gallery mode gyroscopes," Phys. Lett. A 382, 2289-2295 (2018). 


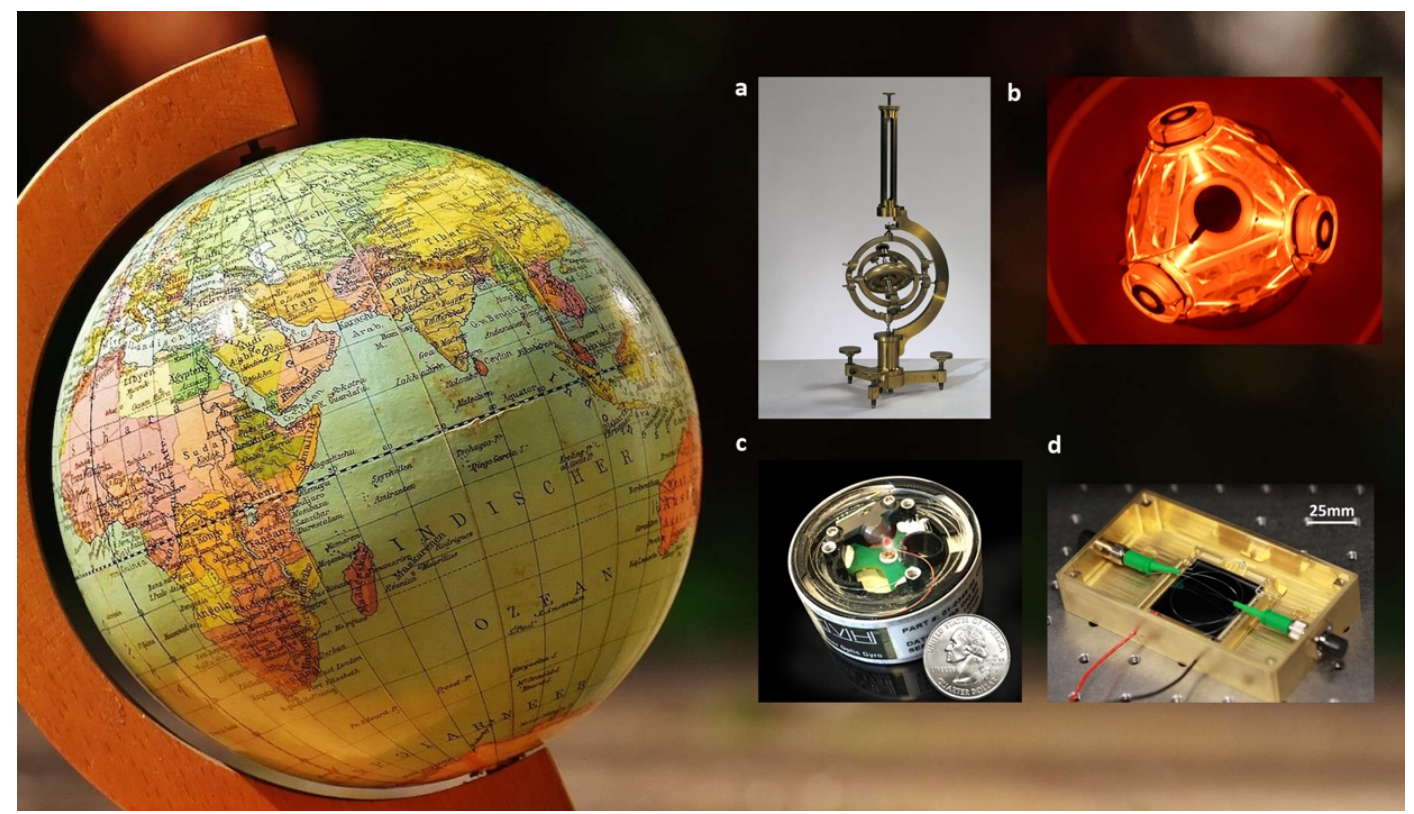

Figure 1 : Brief history of gyroscopes. a, the original spinning mass Foucault gyroscope (70cm height, 1852) [1]. b, the Helium:Neon ring laser gyroscope $(33 \mathrm{~cm}, 1963)$ [4]. c, The interferometric fiber optic gyroscope (10 cm diameter, 1976) [5], the current miniaturized chip-scale Brillouin gyroscope ( $36 \mathrm{~mm}, 2020)$ [9].
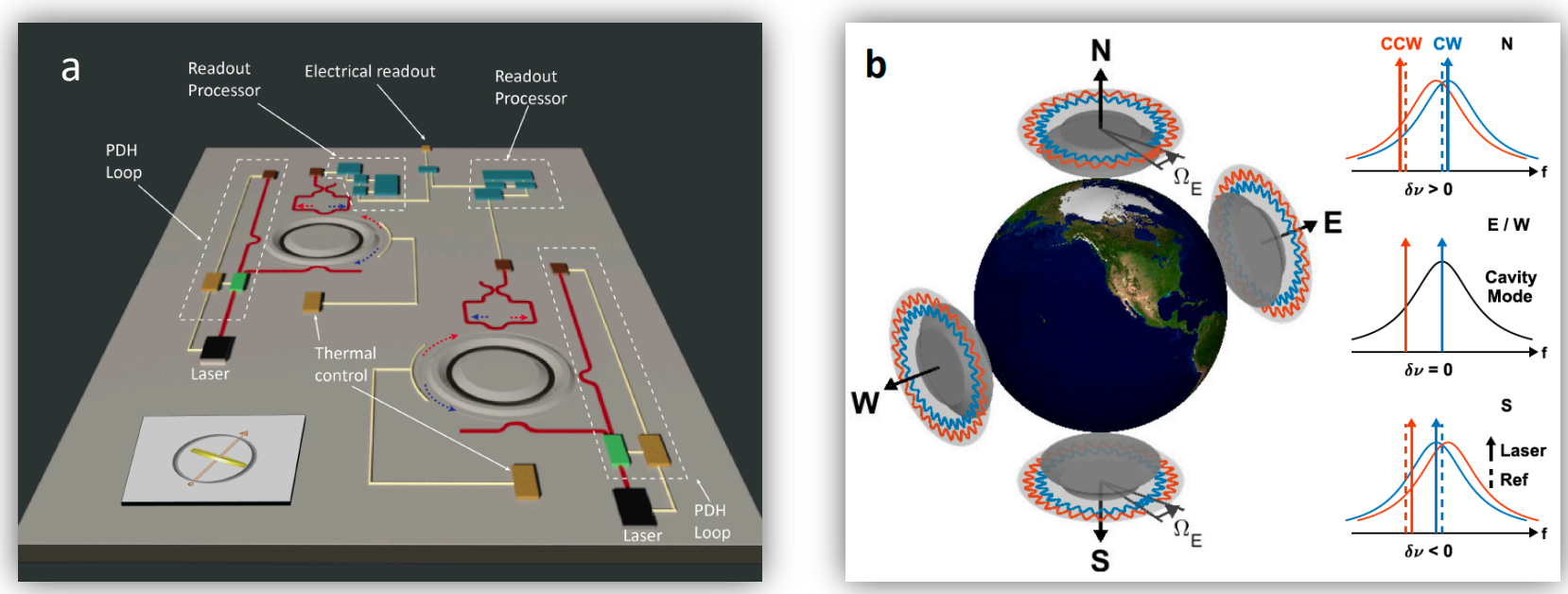

Figure 2 (adapted from ref [10]) : $\mathrm{d}$, Conceptual illustration of the photonic integrated circuit using a waveguideintegrated ultra-high-Q silica resonator on a silicon chip [8]. The blue (red) arrows indicate higher (lower) Brillouin frequency. Red line: optical waveguide, Yellow line: electrical wire, Black: laser, Cyan: acousto-optic modulator, Cyan: controller, Brown: detector, Blue: electronics. b, Operation of the chip Brillouin gyroscope with its surface aligned North and South induces opposite Sagnac shifts, while with the normal axis pointed towards East and West results in no shift. Solid curve: cavity mode. Solid arrows: lasing modes with Sagnac shift. Dashed lines: lasing modes without rotation. CW: clockwise. CCW: counterclockwise. 